\title{
Decreased Systemic Levels of Endocan-1 and CXCL16 in Psoriasis Are Restored following Narrowband UVB Treatment.
}

Gunnthorunn Sigurdardottir, Anna-Karin Ekman, Deepti Verma and Charlotta Enerbäck

The self-archived postprint version of this journal article is available at Linköping University Institutional Repository (DiVA):

http:// urn.kb.se/ resolve?urn=urn:nbn:se:liu:diva-151989

N.B.: When citing this work, cite the original publication.

Sigurdardottir, G., Ekman, A., Verma, D., Enerbäck, C., (2018), Decreased Systemic Levels of Endocan-1 and CXCL16 in Psoriasis Are Restored following Narrowband UVB Treatment., Dermatology. https:// doi.org/ 10.1159/000491819

Original publication available at:

https:// doi.org/ 10.1159/000491819

Copyright: Karger Publishers

http:// www.karger.com/ 


\section{Decreased systemic levels of endocan-1 and CXCL16 in}

psoriasis are restored following narrow-band UVB

\section{treatment}

G. Sigurdardottir ${ }^{1}$; A-K. Ekman ${ }^{1}$; D. Verma ${ }^{1}$; C. Enerbäck ${ }^{1}$

${ }^{1}$ Ingrid Asp Psoriasis Research Center, Department of Clinical and Experimental Medicine,

Division of Cell Biology and Dermatology, Faculty of Medicine and Health Sciences, Linköping University, Linköping, Sweden

Running title: Decreased levels of endocan-1 and CXCL16 in psoriasis

Key words: Psoriasis, UVB, skin, cardiovascular risk

Word count: 2011; Figures: 2; Tables: 1

Key message: Decreased systemic levels of cardiovascular markers endocan-1 and CXCL16 are restored following UVB therapy

Conflicts of interest: The authors have no conflicts of interest

\section{Corresponding author:}

Sigurdardottir, Gunnthorunn,

Department of Clinical and Experimental Medicine, Division of Cell Biology and Dermatology, Linköping University,

SE-581 85 Linköping, Sweden

Phone +46101037429

E-mail: gunnthorunn.sigurdardottir@regionostergotland.se 


\section{Abbreviations and acronyms}

NB, narrowband; UVB, ultraviolet B; CXCL, chemokine (C-X-C motif) ligand; CCL, chemokine (C-C motif) ligand; TNF, tumor necrosis factor; WHR, waist-hip ratio; BMI, body mass index; PTX3, pentraxin 3; FABP, fatty acid binding protein; LDL, low density lipoprotein; HDL, high density lipoprotein; HbA1c, hemoglobin A1c; PASI, psoriasis Area Severity Index; sIL-1R, soluble interleukin-1 receptor; sIL-2Ra, soluble interleukin-2 receptor alpha; sTNFR, soluble tumor necrosis factor receptor; sVEGFR, soluble vascular endothelial growth factor receptor; IFN, interferon; LFA, lymphocyte function-associated antigen; ICAM, intercellular adhesion molecule 


\section{Abstract}

Background: In psoriasis, a common immune mediated disease affecting $2-3 \%$ of the population worldwide, there is an increased prevalence of extra-cutaneous diseases including obesity, metabolic syndrome and cardiovascular disease. This is believed to be linked to systemic inflammation. In previous studies, we have explored various markers in plasma and serum to characterize the on-going systemic inflammation in psoriasis patients compared to controls. We have identified several markers that were altered in psoriasis patients, but which all were unresponsive to NB-UVB treatment.

Objective: The objective of the study was to evaluate the effect of NB-UVB treatment on markers of cardiovascular risk and systemic inflammation in psoriasis.

Methods: The levels of 17 potential biomarkers with an association with cardiovascular risk were quantitated in plasma from 37 age- and gender-matched psoriasis patients and controls at baseline, and in 21 psoriasis patients after 12 weeks of NB-UVB treatment to identify a systemic treatment response.

Results: We identify mediators, endocan-1, CXCL16, and sVEGFR1 that are systemically decreased in psoriasis at baseline and FABP3, FABP4 and sIL-1R1 that show normal baseline levels. Upon 10-12 weeks of NB-UVB treatment endocan-1 and CXCL16 are restored to normal levels while sVEGF1, FABP3, FABP4 and sIL1R1 show a significant reduction. Conclusion: The current study expands the number of potential biomarkers in psoriasis by including a greater number and greater variety of mediators, approaching the systemic inflammation from additional vantage points, including soluble immune receptors and adipocyte contribution, to provide a more complete picture of the systemic inflammatory state in psoriasis. 


\section{Introduction}

In psoriasis, a common immune mediated disease affecting 2-3\% of the population worldwide $[1,2]$, there is an increased prevalence of extra-cutaneous diseases including obesity, the metabolic syndrome and cardiovascular disease. An increase in mortality, especially in severe psoriasis, has been identified [3-6], and several studies suggest that psoriasis is an independent risk factor for cardiovascular events [5,7-9]. In fact, psoriasis patients have been shown to be in a so called 'prothrombotic state', implying a disturbance in the homeostasis between coagulation and fibrinolysis along with an increased platelet activity, predisposing for cardiovascular thrombotic events [10,11].

In previous studies, we have explored various markers in plasma and serum to characterize the on-going systemic inflammation in psoriasis patients compared to controls. We have identified several circulating cytokines, chemokines and factors associated with cardiovascular disease that were elevated systemically in psoriasis, but were unresponsive to narrowband (NB)-UVB treatment [12,13].

We here identify additional biomarkers which demonstrate a clear response to $10-12$ weeks of NB-UVB treatment. The baseline levels of endocan-1, CXCL16 and sVEGFR1 were significantly reduced in psoriasis patients compared to controls. Upon NB-UVB treatment, endocan-1 and CXCL16 were normalized to control levels, while sVEGFR1 showed a further decrease. We also observed that FABP3, FABP4 and sIL-1R1, which showed no significant differences between patients and controls at baseline were significantly reduced upon NB-UVB treatment. 


\section{$\underline{\text { Materials and methods }}$}

\section{$\underline{\text { Study design }}$}

The patients and control subjects were recruited from the Departments of Dermatology at the University Hospital in Linköping and Sahlgrenska University Hospital in Gothenburg, Sweden. The participants were examined by a dermatologist who verified the diagnosis of psoriasis. The patients had not received phototherapy or systemic treatment for at least four weeks prior to inclusion in the study. The levels of biomarkers were quantitated in 37 age- and gender- or age, gender-, waist hip ratio (WHR) and body mass index (BMI) matched pairs of patients and controls. 21 patients underwent NB-UVB therapy for 10-12 weeks. Plasma samples were collected before and after the NB-UVB treatment for the analysis of the selected biomarkers. Disease severity was assessed using the Psoriasis Area and Severity Index (PASI).

Measurements of biomarkers for endothelial dysfunction, inflammatory markers in fat tissue, lipid binding proteins together with markers of innate immune reactions

Blood samples were collected from patients and controls in sodium heparin coated CPT tubes. Plasma was collected and stored at $-80 \mathrm{C}$ until analysis. The levels of markers for endothelial dysfunction and prothrombotic state including thrombomodulin, pecam-1, pentraxin (PTX)3 and endocan-1, inflammatory markers produced in fat tissue including the adipokine oncostatin $\mathrm{M}$, and the fatty-acid binding proteins (FABP)3 and FABP4 together with the chemokines CXCL16 and CXCL6 was determined. Measurements of the soluble interleukin receptors (sIL)1R1, sIL-1R2, sIL-2Ra, soluble TNF receptors sTNFR1, sTNFR2 and soluble vascular endothelial growth factor receptors (VEGFR) 1, sVEGFR2 and sVEGFR3 were performed. The levels of the selected biomarkers (table 1) were determined using multianalyte profiling from Milliplex ${ }^{\circledR}$ MAP (Millipore Corporation, Billerica, MA, USA), according to the manufacturer's instructions. Sample data were collected on a Luminex 200 instrument 
(Biosource, Nivelles, Belgium) using the xPONENT software (Luminexcorp, Austin, TX) and analyzed in_MasterPlex QT (MiraiBio, Alameda, CA).

\section{Measurement of clinical parameters of metabolic, heart- and vascular disease}

The levels of glucose and hemoglobin (Hb)A1c were measured and the lipid profile (total cholesterol, low density lipoprotein (LDL)- and high density lipoprotein (HDL)-cholesterols, triglycerides and apolipoproteins A1 and B) was characterized according to standard clinical procedures of the Department of Clinical Chemistry at the University Hospital in Linköping, Sweden.

\section{$\underline{\text { Statistical analysis }}$}

Data analysis was performed in GraphPad Prism ${ }^{\circledR}$ version 6.01 (GraphPad Software Inc., San Diego, CA, USA). Data were compared using Mann-Whitney or Wilcoxon matched-pair signed rank test. Correlations were determined by Spearman's test. A p-value of less than or equal to 0.05 was considered significant. 


\section{$\underline{\text { Results }}$}

\section{$\underline{\text { Clinical parameters of metabolic and vascular disease }}$}

The psoriasis patients displayed a significantly higher BMI (median BMI 28.8, range 20.0-37.5) compared to the controls (median BMI 25.0, range 20.8-30.1), $\mathrm{p}=0.01$. The WHR was also significantly higher in the patients (median 0.98, range 0.74-1.26) compared to the controls (median 0.90, range 0.68-1.06), $\mathrm{p}=0.02$. Measurements of total cholesterol, LDL and HDL cholesterols, triglycerides and apolipoproteins A1 and B together with blood glucose and HbA1c did not differ between the psoriasis patients and the controls

\section{Levels of potential biomarkers in psoriasis}

Measurement of a wide array of biomarkers of cardiovascular risk and systemic inflammation in 35-37 age-and gender matched patients and control was performed. The median baseline PASI score for the psoriasis patients was 7.5 (range 1.2-25.3). The level of endocan- 1 was found to be markedly reduced in psoriasis patients compared to controls ( $p=0.009$; Fig. 1a). Interestingly, endocan-1 also showed a negative correlation to PASI ( $r=-0.3, p=0.045)$, with an increasing severity correlating with lower endocan-1 levels. Furthermore, the level of the chemokine CXCL16 was decreased in psoriasis patients compared to the controls ( $p=0.02$; Fig. 1b). When matched for WHR and BMI, these differences receded.

We found no statistically significant difference in the baseline levels of thrombomodulin, pecam-1, PTX3, oncostatin M, FABP3, FABP4 or CXCL6 between psoriasis patients and control subjects.

Among the soluble receptors, we detected reduced levels of sVEGFR1 in the patients ( $\mathrm{p}=0.05$; Fig. 1c). No other significant differences could be detected between the patients and the controls in soluble interleukin, TNF or vascular endothelial growth factor receptors, 


\section{The influence of NB-UVB therapy on biomarker levels}

We compared the levels of the biomarkers before and after 10-12 weeks of NB-UVB therapy in psoriasis patients. The levels of endocan-1 and CXCL16, which were both reduced at baseline in psoriasis patients, were normalized to control levels after the UVB therapy $(p=0.008$ and $p=0.002$, respectively; Fig. $2 a$ and $b$ ). In the same patients, the NB-UVB therapy further reduced the already decreased levels of sVEGFR1 ( $p=0.04$; Fig. 2c).

Furthermore, FABP3, FABP4 and sIL-1R1, which had similar levels in patients and controls at baseline demonstrated a significant decrease in the patients upon NB-UVB treatment $(p=0.03$, $p=0.04$ and $p=0.005$, respectively; Fig. 2d, e and f).

There was a significant reduction in PASI scores after the UV treatment, median PASI score before treatment being 8.3 (range 2.2-17.2) and after treatment 1.8 (range 0.0-5.6), $\mathrm{p}<0.0001$. 


\section{$\underline{\text { Discussion }}$}

It has become increasingly evident that many immune-mediated diseases share an increased risk for cardiovascular disease. Inflammatory mechanisms affect the homeostasis of the vascular endothelium, leading to an endothelial dysfunction locally in the targeted tissue and often also systemically. Finding biomarkers that not only reflect the psoriasis disease state but are also indicative of associated comorbidities would be highly valuable.

In this study, we analyzed the levels of a multitude of potential biomarkers that are also markers of cardiovascular risk, to investigate their suitability as biomarker and whether they can be used as a quantitation of the treatment response to UVB. In doing so, we identified three mediators whose expression was reduced in psoriasis: endocan-1, CXCL16 and sVEGFR1.

Interestingly, while the biomarkers we have previously explored in psoriasis tended to remain unaffected by NB-UVB therapy, we here observe that NB-UVB therapy normalizes the levels of endocan-1 and CXCL16 to the levels of the control subjects, and further reduces the levels of sVEGFR1. This altered response indicates that these mediators might be susceptible enough to treatment to properly identify and characterize the response.

Endocan-1 has previously been linked to endothelial dysfunction, a hallmark of atherosclerosis, and is being explored as a prognostic factor/biomarker in sepsis, cancer and cardiovascular disease. Under normal physiological conditions, endocan-1 is vasoprotective, but the levels of endocan-1 are increased in many inflammatory and hypervascular states, perhaps as a protective response. Low levels may therefore pinpoint to diminished vasoprotective effects [14-17]. Furthermore, decreased endocan-1 levels are described in overweight and non-alcoholic fatty liver disease (NAFLD), conditions overexpressed in psoriasis [17-20].

The reduction of endocan-1 that we observe is also interesting in the context of leukocyte extravasation, where endocan-1 inhibits the extravasation process by preventing the binding of leukocyte function-associated antigen (LFA)-1 to intracellular adhesion molecule (ICAM)-1 
[21]. Interestingly, we have previously found elevated levels of soluble ICAM-1 in this psoriasis population [13]. It may be speculated that the reduced endocan-1 levels may be the result of a feedback loop stemming from the increased levels of ICAM-1, and that the low levels of endocan-1 may result in a lack of inhibition of the leukocyte transmigratory responses. Such a mechanism has also been proposed in atopic dermatitis [22].

Previous studies on endocan-1 in psoriasis are contradictory. Balta et al have identified elevated levels of endocan-1 in psoriasis, which also correlated positively to disease severity [23]. However, this elevation was not replicated by Toprak et al who found no differences in endocan-1 levels between psoriasis patients and controls [24]. This emphasizes that the role of endocan-1 in psoriasis is uncertain and not be fully explored.

We also found notably lower levels of CXCL16 in psoriasis patients than in controls. CXCL16 is produced constitutively by keratinocytes and expressed by macrophages in atherosclerosis lesions. The relationship between circulating level of soluble CXCL16 and atherosclerotic disorders remains controversial in clinical practice. Both decreased and increased CXCL16 levels have been reported in patients with atherosclerotic disorders [25-27]. CXCL16 is increased in the lesional psoriatic skin, where it is most prominent in the lower epidermis [28]. Compared to healthy and atopic dermatitis skin, monocytes, keratinocytes and dendritic cells in the psoriatic skin express a higher level of CXCL16 [29]. In contrast, previous studies on the systemic levels of CXCL16 found no increase in the serum of psoriasis patients, and no correlation between CXCL16 levels and disease severity [30].

Our measurements of soluble receptors in psoriasis revealed decreased levels of the sVEGFR1. sVEGFR1 binds and sequesters VEGF, acting as an endogenous inhibitor of the VEGF response [31]. This is particularly interesting in the context of psoriasis, where VEGF is over-expressed both in the lesional skin and in serum [32]. sVEGFR1 is labelled a cardiovascular risk marker because of its association with endothelial dysfunction [33]. The 
reduced levels of sVEGFR1 in plasma samples from psoriasis patients at baseline, along with the further reduction after NB-UVB, may illustrate the dysfunctional VEGF pathway in psoriasis and an insufficient inhibition of VEGF that despite the reduction of sVEGFR1 is rendered less noticeable after UVB due to the attenuating effect of UVB also on VEGF levels [34]. In serum from psoriasis patients with mild disease, Flisiak et al reported higher baseline level of sVEGF1, which further increased upon topical treatment [35].

FABPs are key players in lipid homeostasis and comprise several isoforms. The heart and adipocyte isoforms FABP3-4, have been well studied in other systemic disorders but not fully investigated in psoriasis. FABP3 is highly expressed in the cardiac and skeletal muscles and its presence in blood is indicative of acute myocardial infarction [36]. FABP4, an adipokine and the most commonly occurring FABP isoform, is linked to obesity, diabetes, NALFD and atherosclerosis $[37,38]$

We did not observe increased baseline levels of either FABP3 or FABP4 in patients compared to controls. However, NB-UVB treatment led to a significant reduction in both FABP3 and FABP4 levels suggesting an influence of UVB on lipid metabolism through the above isoforms. Our results are partially in agreement with a recently published paper by Baran et al [39], reporting no differences in baseline levels of FABP3 in patients and controls. In contrast to our results, they report increased baseline FABP4 levels in the patients which does not alter upon topical treatment.

In this study, we have screened a large number of putative biomarkers for the systemic inflammation in psoriasis. They were selected based on previous association to the risk of cardiovascular disease. Numerous studies have been performed with the aim of establishing soluble biomarkers for the systemic inflammation in psoriasis. However, most of the biomarkers that have been studied have not succeeded in meeting the criteria for a clinically useful biomarker. This work contributes by the evaluation of a larger number of candidate 
biomarkers in psoriasis. It is apparent that further studies are needed to elucidate the role of the investigated biomarkers in the pathogenesis of psoriasis and its comorbidities. 


\section{Compliance with ethical standards}

\section{Conflict of interest}

The authors declare that they have no conflict of interest

\section{$\underline{\text { Ethical approval }}$}

Written informed consent was obtained from the patients and control subjects, and the ethical principles of the Declaration of Helsinki were followed. The study was approved by the local ethics committee.

\section{Funding sources}

This research was funded by the Ingrid Asp Foundation, the Welander Foundation, the Swedish Psoriasis Association and the Medical Research Council.

\section{Acknowledgements}

The research was supported by funding from the Ingrid Asp Foundation, the Welander Foundation, the Swedish Psoriasis Association and the Medical Research Council. 


\section{$\underline{\text { Tables }}$}

Table 1. The evaluated markers

\begin{tabular}{ll}
\hline Mediator, full name & Abbreviated name \\
\hline $\begin{array}{l}\text { Chemokine (CXC-motif) ligand 6 } \\
\text { Chemokine (CXC-motif) ligand 16 }\end{array}$ & CXCL6 \\
$\begin{array}{l}\text { Endocan-1 } \\
\text { Fatty acid binding protein 3 }\end{array}$ & \\
Fatty acid binding protein 4 & FABP3 \\
Oncostatin M & FABP4 \\
Pecam-1 & OSM \\
$\begin{array}{l}\text { Pentraxin 3 } \\
\text { Trombomodulin }\end{array}$ & \\
soluble Interleukin 1 Receptor type 1 & PTX3 \\
soluble Interleukin 1 Receptor type 2 & \\
soluble Interleukin 2 Receptor subunit alpha & sIL-1R1 \\
soluble Tumor Necrosis Factor Receptor 1 & sIL-1R2 \\
soluble Tumor Necrosis Factor Receptor 2 & sIL-2Ra \\
soluble Vascular Endothelial Growth Factor Receptor 1 & sVEGFR1 \\
soluble Vascular Endothelial Growth Factor Receptor 2 & sVEGFR2 \\
soluble Vascular Endothelial Growth Factor Receptor 3 & sVEGFR3 \\
\hline
\end{tabular}




\section{Figure legends}

Fig. 1. Baseline levels of (a) endocan-1 ( $n=35)$, (b) CXCL16 ( $n=35$ ) and (c) sVEGFR1( $n=37)$ measured systemically in age-and gender matched psoriasis patients (psoriasis) and control subjects (controls). The line shows the median level. ${ }^{*} \mathrm{p} \leq 0.05,{ }^{* *} \mathrm{p}<0.01$.

Fig. 2. The levels of (a) endocan-1, (b) CXCL16, (c) sVEGFR1, (d) FABP3, (e) FABP4 $(\mathrm{n}=21)$ and (f) IL-1R1 $(\mathrm{n}=15)$ in the plasma of psoriasis patients before and after NB-UVB therapy. ${ }^{*} \mathrm{p} \leq 0.05,{ }^{* *} \mathrm{p}<0.01$. 


\section{References}

1 Langley RG, Krueger GG, Griffiths CE: Psoriasis: epidemiology, clinical features, and quality of life. Annals of the rheumatic diseases 2005;64 Suppl 2:ii18-23; discussion ii24-15.

2 Parisi R, Symmons DP, Griffiths CE, Ashcroft DM: Global epidemiology of psoriasis: a systematic review of incidence and prevalence. The Journal of investigative dermatology 2013;133:377-385.

3 Menter A, Griffiths CE, Tebbey PW, Horn EJ, Sterry W: Exploring the association between cardiovascular and other disease-related risk factors in the psoriasis population: the need for increased understanding across the medical community. Journal of the European Academy of Dermatology and Venereology : JEADV 2010;24:1371-1377.

4 Gelfand JM, Troxel AB, Lewis JD, Kurd SK, Shin DB, Wang X, Margolis DJ, Strom BL: The risk of mortality in patients with psoriasis: results from a population-based study. Archives of dermatology 2007;143:1493-1499.

5 Prodanovich S, Kirsner RS, Kravetz JD, Ma F, Martinez L, Federman DG: Association of psoriasis with coronary artery, cerebrovascular, and peripheral vascular diseases and mortality. Archives of dermatology 2009;145:700-703.

6 Ahlehoff O, Gislason GH, Charlot M, Jorgensen CH, Lindhardsen J, Olesen JB, Abildstrom SZ, Skov L, Torp-Pedersen C, Hansen PR: Psoriasis is associated with clinically significant cardiovascular risk: a Danish nationwide cohort study. Journal of internal medicine 2011;270:147-157.

7 Gelfand JM, Dommasch ED, Shin DB, Azfar RS, Kurd SK, Wang X, Troxel AB: The risk of stroke in patients with psoriasis. The Journal of investigative dermatology 2009;129:2411-2418.

8 Gelfand JM, Neimann AL, Shin DB, Wang X, Margolis DJ, Troxel AB: Risk of myocardial infarction in patients with psoriasis. JAMA : the journal of the American Medical Association 2006;296:1735-1741.

9 Mehta NN, Azfar RS, Shin DB, Neimann AL, Troxel AB, Gelfand JM: Patients with severe psoriasis are at increased risk of cardiovascular mortality: cohort study using the General Practice Research Database. European heart journal 2010;31:1000-1006.

10 Davidovici BB, Sattar N, Prinz J, Puig L, Emery P, Barker JN, van de Kerkhof P, Stahle M, Nestle FO, Girolomoni G, Krueger JG: Psoriasis and systemic inflammatory diseases: potential mechanistic links between skin disease and co-morbid conditions. The Journal of investigative dermatology 2010;130:1785-1796.

11 Dyring-Andersen B, Geisler C, Agerbeck C, Lauritsen JP, Gudjonsdottir SD, Skov L, Bonefeld CM: Increased number and frequency of group 3 innate lymphoid cells in nonlesional psoriatic skin. The British journal of dermatology 2014;170:609-616.

12 Ekman AK, Sigurdardottir G, Carlstrom M, Kartul N, Jenmalm MC, Enerback C: Systemically elevated Th1-, Th2- and Th17-associated chemokines in psoriasis vulgaris before and after ultraviolet B treatment. Acta dermato-venereologica 2013;93:527-531.

13 Sigurdardottir G, Ekman AK, Stahle M, Bivik C, Enerback C: Systemic treatment and narrowband ultraviolet $\mathrm{B}$ differentially affect cardiovascular risk markers in psoriasis. $\mathrm{J}$ Am Acad Dermatol 2014;70:1067-1075.

$14 \quad$ Balta S, Mikhailidis DP, Demirkol S, Celik T, Ozturk C, Iyisoy A: Endocan and atherosclerosis. Angiology 2015;66:490.

15 Balta S, Mikhailidis DP, Demirkol S, Ozturk C, Celik T, Iyisoy A: Endocan: A novel inflammatory indicator in cardiovascular disease? Atherosclerosis 2015;243:339-343.

16 Sarrazin S, Adam E, Lyon M, Depontieu F, Motte V, Landolfi C, Lortat-Jacob H, Bechard D, Lassalle P, Delehedde M: Endocan or endothelial cell specific molecule-1 (ESM- 
1): a potential novel endothelial cell marker and a new target for cancer therapy. Biochimica et biophysica acta 2006;1765:25-37.

17 Janke J, Engeli S, Gorzelniak K, Feldpausch M, Heintze U, Bohnke J, Wellner M, Herse F, Lassalle P, Luft FC, Sharma AM: Adipose tissue and circulating endothelial cell specific molecule-1 in human obesity. Hormone and metabolic research $=$ Hormon- und Stoffwechselforschung $=$ Hormones et metabolisme 2006;38:28-33.

18 Jensen P, Skov L: Psoriasis and Obesity. Dermatology 2017

19 Tok D, Ekiz F, Basar O, Coban S, Ozturk G: Serum endocan levels in patients with chronic liver disease. International journal of clinical and experimental medicine 2014;7:1802-1807.

20 Candia R, Ruiz A, Torres-Robles R, Chavez-Tapia N, Mendez-Sanchez N, Arrese M: Risk of non-alcoholic fatty liver disease in patients with psoriasis: a systematic review and meta-analysis. Journal of the European Academy of Dermatology and Venereology : JEADV 2015;29:656-662.

21 Bechard D, Scherpereel A, Hammad H, Gentina T, Tsicopoulos A, Aumercier M, Pestel J, Dessaint JP, Tonnel AB, Lassalle P: Human endothelial-cell specific molecule-1 binds directly to the integrin CD11a/CD18 (LFA-1) and blocks binding to intercellular adhesion molecule-1. Journal of immunology 2001;167:3099-3106.

22 Suzuki H, Miyagaki T, Otobe S, Nakajima R, Oka T, Takahashi N, Kabasawa M, Suga H, Yoshizaki A, Asano Y, Sato S, Sugaya M: Increased endocan expression in lesional skin and decreased endocan expression in sera in atopic dermatitis. The Journal of dermatology 2017;44:1392-1395.

23 Balta I, Balta S, Demirkol S, Mikhailidis DP, Celik T, Akhan M, Kurt O, Kurt YG, Aydin I, Kilic S: Elevated serum levels of endocan in patients with psoriasis vulgaris: correlations with cardiovascular risk and activity of disease. The British journal of dermatology 2013;169:1066-1070.

24 Erek Toprak A, Ozlu E, Uzuncakmak TK, Yalcinkaya E, Sogut S, Karadag AS: Neutrophil/Lymphocyte Ratio, Serum Endocan, and Nesfatin-1 Levels in Patients with Psoriasis Vulgaris Undergoing Phototherapy Treatment. Medical science monitor : international medical journal of experimental and clinical research 2016;22:1232-1237.

25 Tohyama M, Sayama K, Komatsuzawa H, Hanakawa Y, Shirakata Y, Dai X, Yang L, Tokumaru S, Nagai H, Hirakawa S, Sugai M, Hashimoto K: CXCL16 is a novel mediator of the innate immunity of epidermal keratinocytes. International immunology 2007;19:1095-1102.

26 Aslanian AM, Charo IF: Targeted disruption of the scavenger receptor and chemokine CXCL16 accelerates atherosclerosis. Circulation 2006;114:583-590.

27 Ma A, Pan X, Xing Y, Wu M, Wang Y, Ma C: Elevation of serum CXCL16 level correlates well with atherosclerotic ischemic stroke. Archives of medical science : AMS 2014;10:47-52.

28 Oh ST, Schramme A, Tilgen W, Gutwein P, Reichrath J: Overexpression of CXCL16 in lesional psoriatic skin. Dermato-endocrinology 2009;1:114-118.

29 Gunther C, Carballido-Perrig N, Kaesler S, Carballido JM, Biedermann T: CXCL16 and CXCR6 are upregulated in psoriasis and mediate cutaneous recruitment of human CD8+ T cells. The Journal of investigative dermatology 2012;132:626-634.

30 Lima XT, Oliveira RT, Braga FG, Magalhaes RF, Mamoni RL, Blotta MH: Circulating levels of chemokines in psoriasis. Autoimmunity 2015;48:57-60.

31 Kendall RL, Wang G, Thomas KA: Identification of a natural soluble form of the vascular endothelial growth factor receptor, FLT-1, and its heterodimerization with KDR. Biochemical and biophysical research communications 1996;226:324-328. 
Canavese M, Altruda F, Ruzicka T, Schauber J: Vascular endothelial growth factor (VEGF) in the pathogenesis of psoriasis--a possible target for novel therapies? Journal of dermatological science 2010;58:171-176.

33 Wu FT, Stefanini MO, Mac Gabhann F, Kontos CD, Annex BH, Popel AS: A systems biology perspective on sVEGFR1: its biological function, pathogenic role and therapeutic use. Journal of cellular and molecular medicine 2010;14:528-552.

34

Chen HQ, Li X, Tang R: Effects of Narrow Band Ultraviolet B on Serum Levels of Vascular Endothelial Growth Factor and Interleukin-8 in Patients with Psoriasis. American journal of therapeutics 2016;23:e655-662.

35 Flisiak I, Zaniewski P, Rogalska-Taranta M, Chodynicka B: Effect of psoriasis therapy on VEGF and its soluble receptors serum concentrations. J Eur Acad Dermatol Venereol 2012;26:302-307.

36 Smathers RL, Petersen DR: The human fatty acid-binding protein family: evolutionary divergences and functions. Hum Genomics 2011;5:170-191.

$37 \quad$ Furuhashi M, Saitoh S, Shimamoto K, Miura T: Fatty Acid-Binding Protein 4 (FABP4): Pathophysiological Insights and Potent Clinical Biomarker of Metabolic and Cardiovascular Diseases. Clinical Medicine Insights Cardiology 2014;8:23-33.

38 Hotamisligil GS, Bernlohr DA: Metabolic functions of FABPs--mechanisms and therapeutic implications. Nature reviews Endocrinology 2015;11:592-605.

39 Baran A, Swiderska M, Bacharewicz-Szczerbicka J, Mysliwiec H, Flisiak I: Serum Fatty Acid-Binding Protein 4 is Increased in Patients with Psoriasis. Lipids 2017;52:5160. 

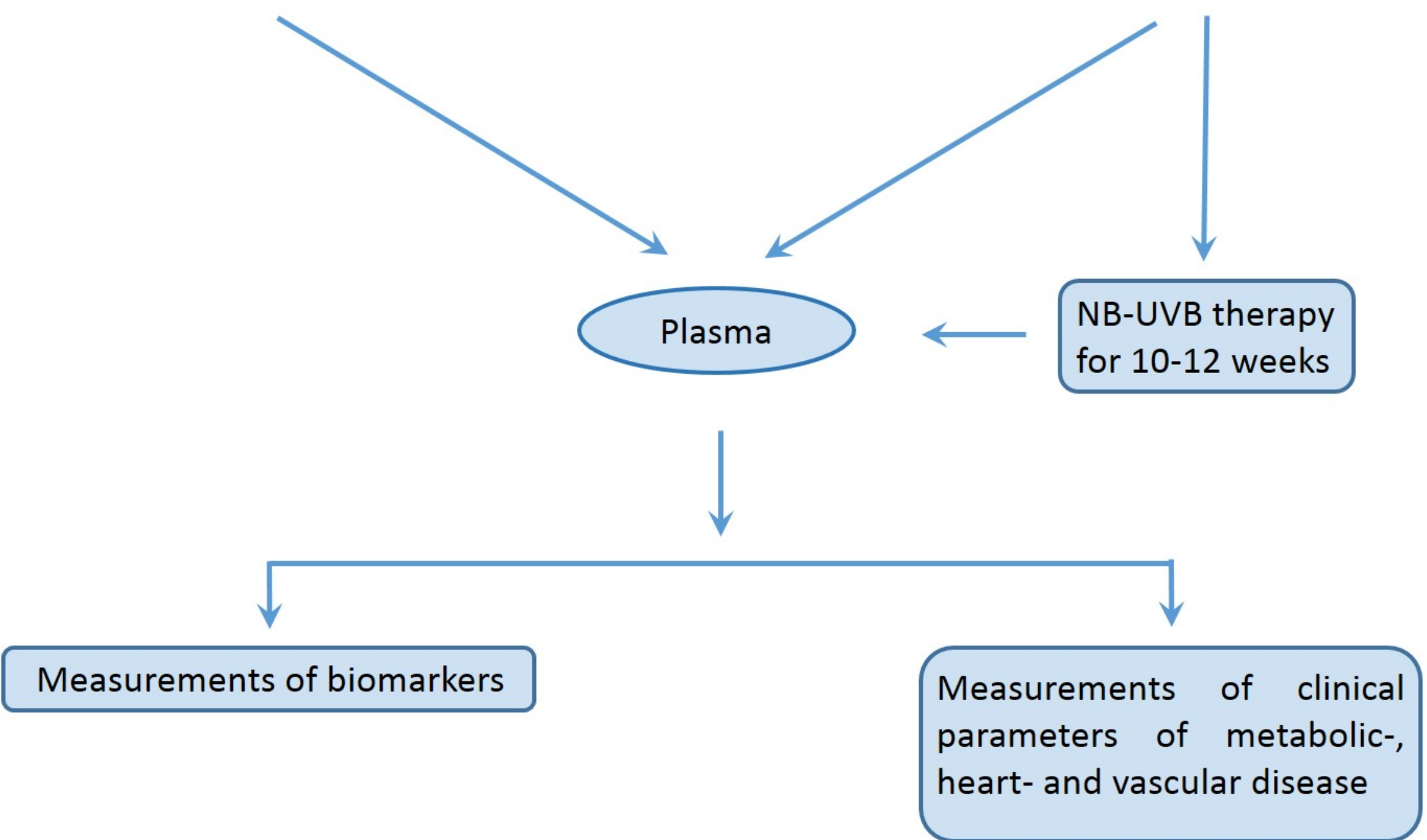
(a)

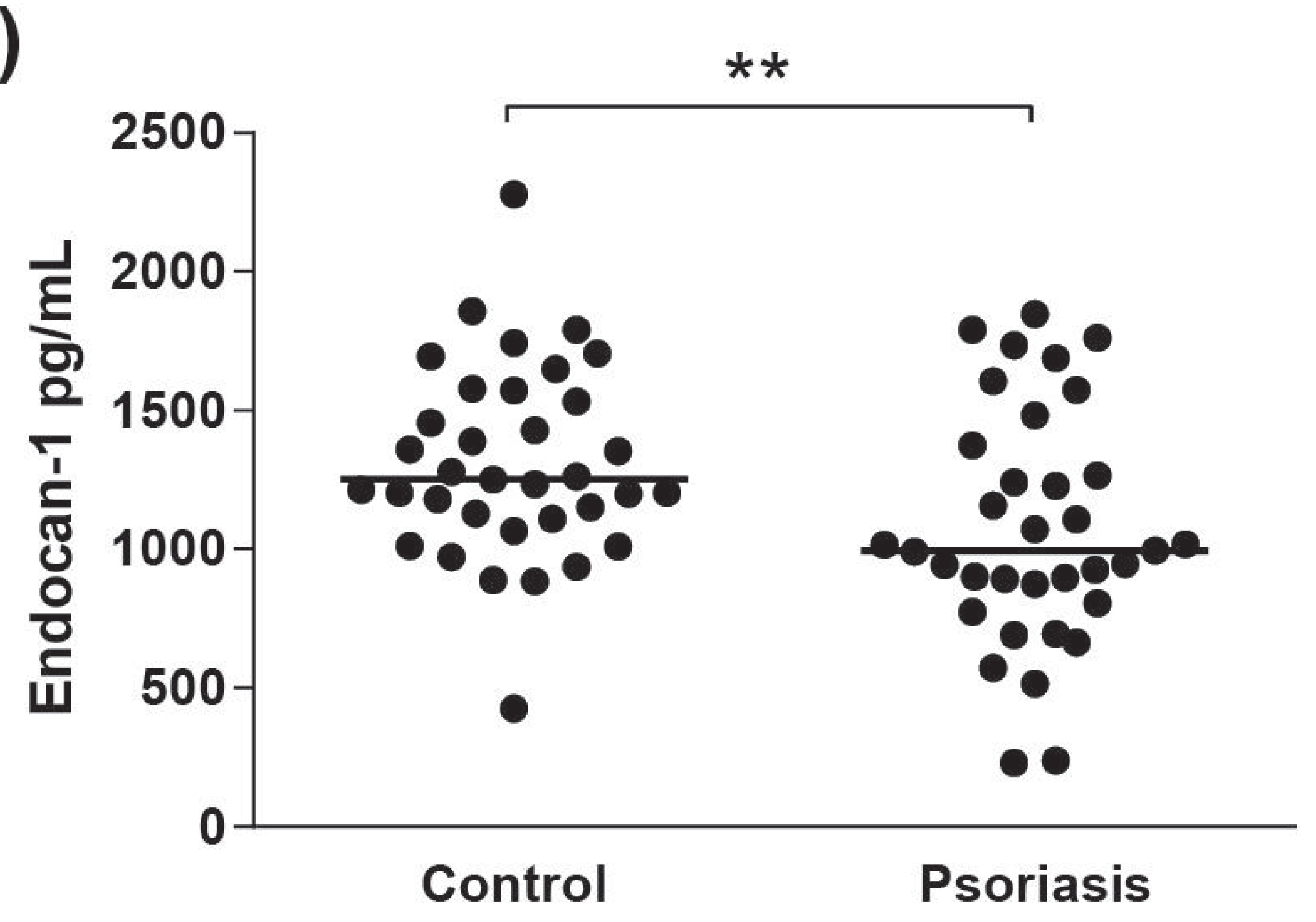

(b)

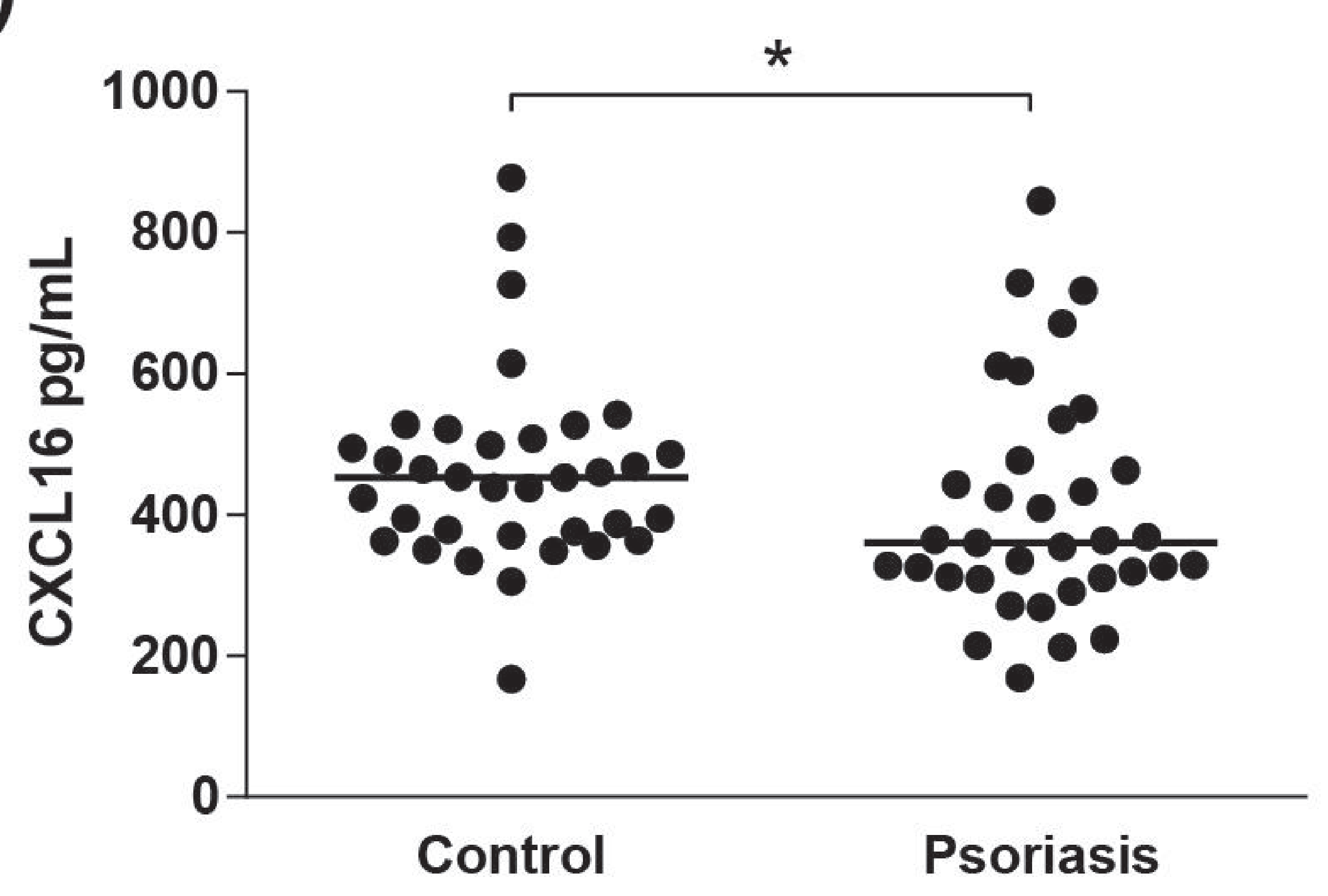

(c)

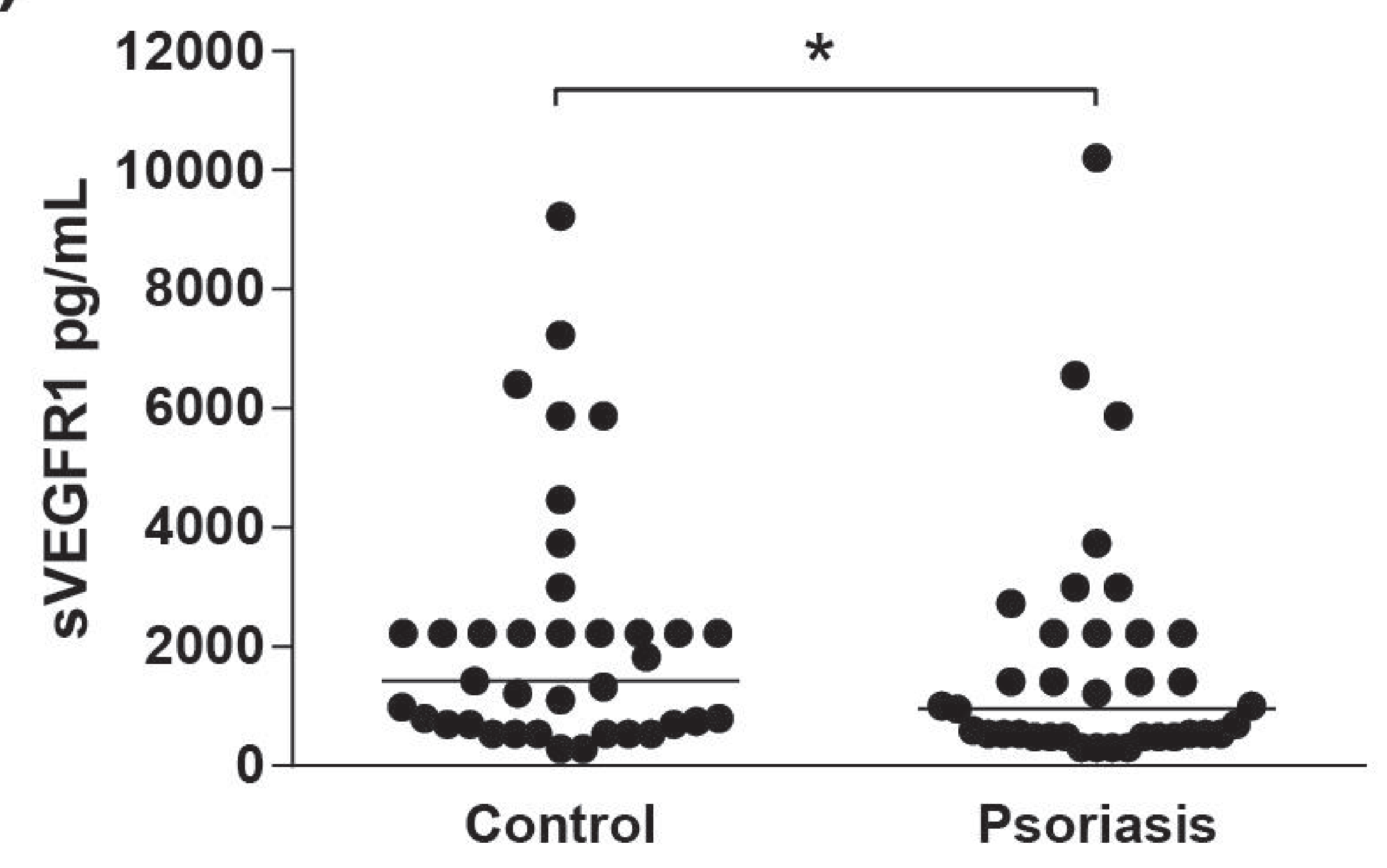


(a)

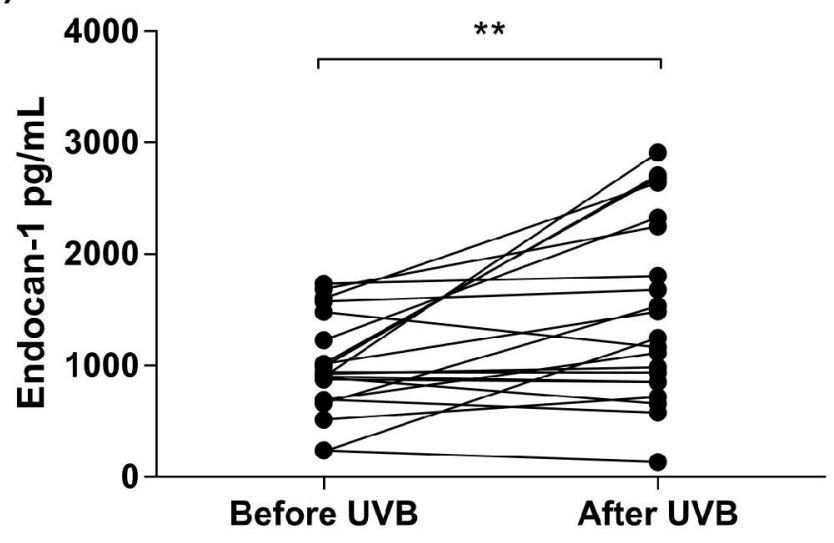

(c)

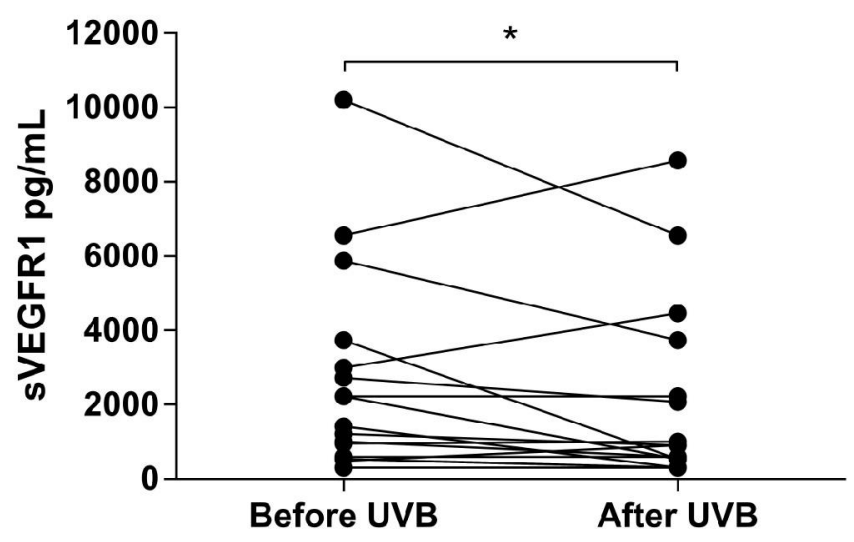

(e)

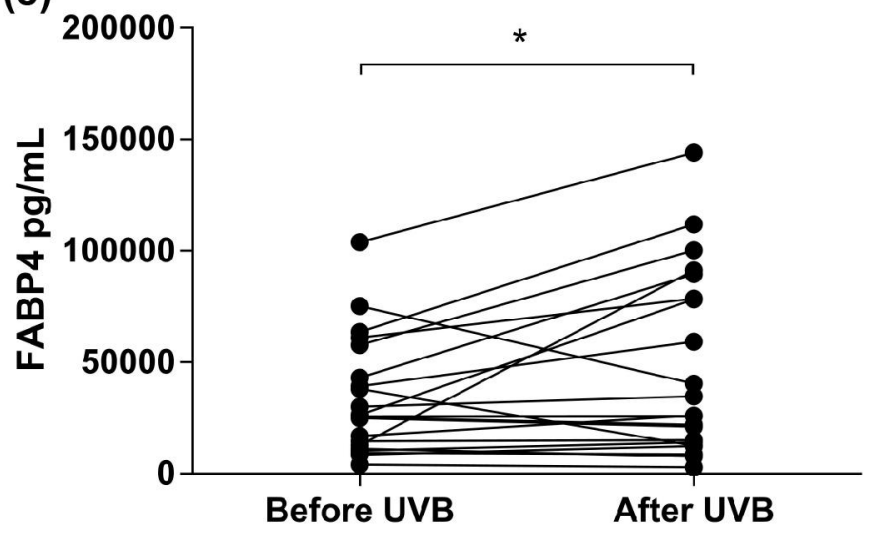

(b)

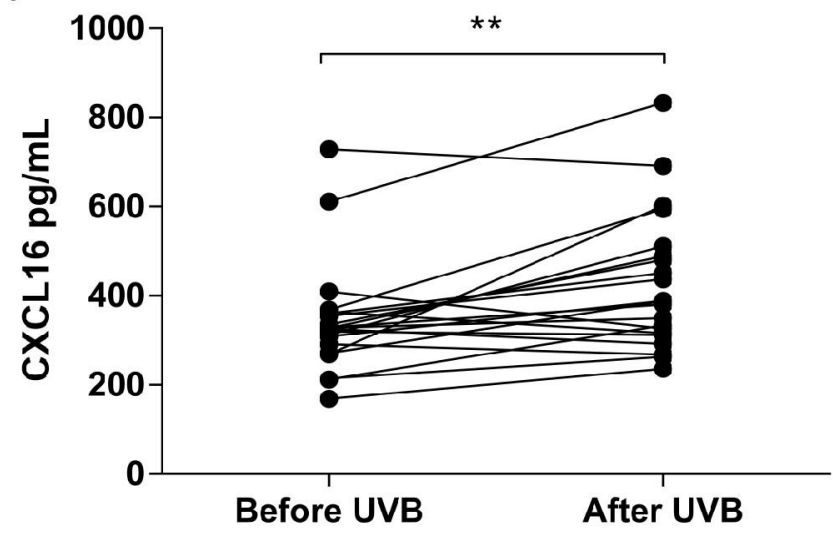

(d)

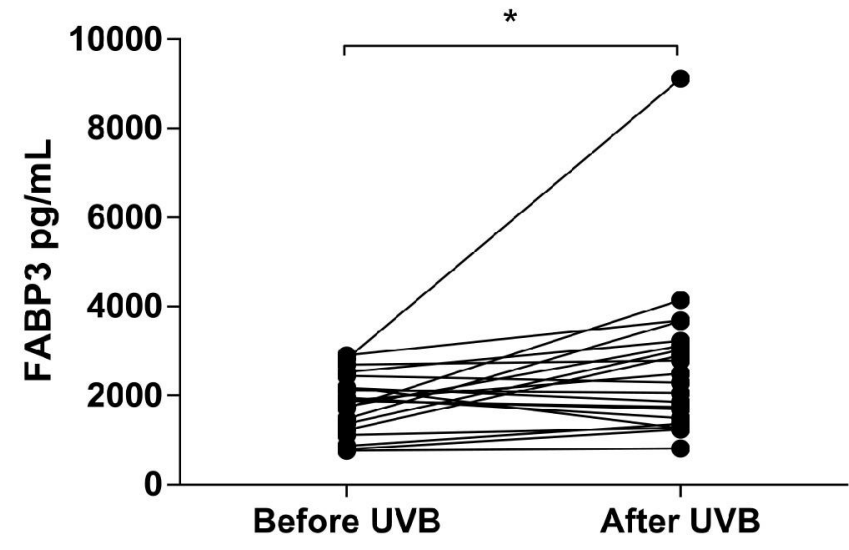

(f)

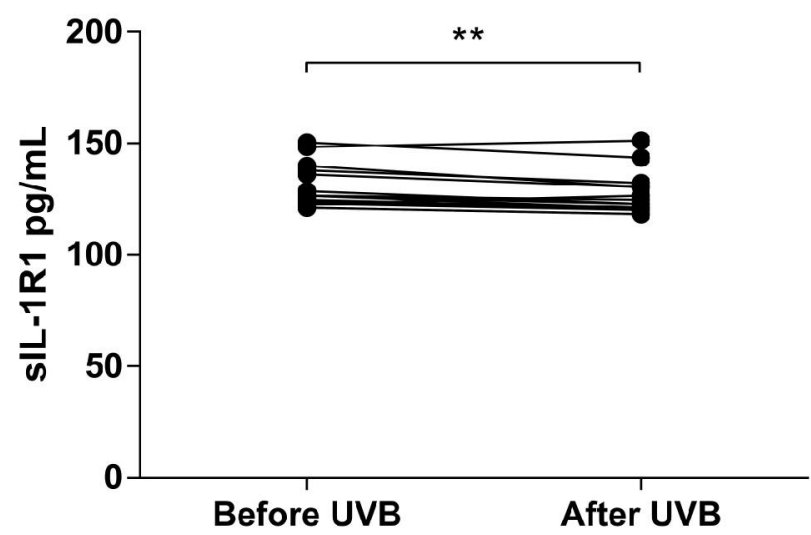

